\title{
Chronic Pain Review Following Lichtenstein Hernia Repair: A Personal Series
}

\author{
Maurice Brygel $^{*}$, Luke J. Bonato ${ }^{2}$, Sam S. Farah ${ }^{3,4}$ \\ ${ }^{1}$ The Melbourne Hernia Clinic, Masada Hospital, Melbourne, Australia \\ ${ }^{2}$ Surgical Assistant, Master of Surgery Student, Monash University, Melbourne, Australia \\ ${ }^{3}$ Surgical Resident, Southern Health, Melbourne Australia \\ ${ }^{4}$ Master of Medicine (Clinical Epidemiology) Student, University of Sydney, Sydney, Australia \\ Email: *mbrygel@netspace.net.au
}

Received July 16, 2012; revised August 17, 2012; accepted August 31, 2012

\begin{abstract}
Introduction: Chronic groin pain is both a topical subject and important outcome measurement following inguinal hernia repair. It has been suggested its incidence is related to the management of the nerves of the inguinal canal as well as the type of mesh used and methods of fixation for both open and laparoscopic surgery. The level of pre-operative and post operative pain, its duration as well as complications may all be factors in predicting whether chronic pain could develop. The method of measurement of chronic pain is itself a contentious issue. It is now apparent that the qualitative measurement of activity and functional status as well as quantitative is important. As a result several uniform methods of assessing chronic post-operative pain have been designed, validated and implemented. One of these is used here. Methods: A study reviewing a consecutive series of Lichtenstein repairs performed by a single experienced hernia surgeon was carried out. 248 inguinal hernia patients operated on in 2005 were reviewed. Patients were contacted via telephone at a median of 50 months. Franneby's recently validated inguinal pain questionnaire was used to assess the incidence of chronic pain. Results: 185 (75\%) patients were able to be contacted for follow-up, making a total of 213 inguinal hernia repairs (including bilateral and recurrent hernias). At the time of review 3\% of patients reported having pain. No patients reported that pain or discomfort was limiting their work, exercise or activities of daily living. No patients had disabling pain. Conclusion: Chronic pain did not appear to be a major problem within this cohort of patients. The Lichtenstein technique can produce favourable results in terms of chronic pain for unilateral, bilateral and recurrent inguinal hernias in an unselected group of patients with the usual mix of risk factors and complications.
\end{abstract}

Keywords: Inguinal Hernia; Lichtenstein; Local Anaesthesia; Chronic Pain; Bilateral Inguinal Hernia; Recurrent Inguinal Hernia

\section{Introduction}

Inguinal hernia repairs are one of the most common surgical procedures [1]. The pre-eminent status of the original Lichtenstein technique has been challenged with the introduction of other open and laparoscopic techniques, lightweight meshes and new methods of fixation with absorbable tackers and tissue glues. While there has been significant improvement in recurrence rates with most types of mesh repair [2], a variable and worrying incidence of chronic pain following open and laparoscopic repair of inguinal hernias has been documented [3].

There is still controversy regarding the true incidence of chronic pain. The lack of uniform definitions and interpretation as well as different methods of assessment has lead to this [4-6]. Mild, moderate and severe pain has been reported to have a prevalence of $0.7 \%$ to $43.3 \%$ [3],

\footnotetext{
*Corresponding author.
}

with some treating the presence of pain as a dichotomous (yes/no) entity [7]. An overall prevalence of $0.5 \%$ - 6\% of severe debilitating pain affecting normal daily activities and work has been reported [3]. It has also been suggested that the rates of severe chronic pain are lower with laparoscopic repair, compared with Lichtenstein repair or other open techniques, as well as being associated with earlier return to work and normal activities [8,9]. This however is associated with more adverse events during surgery [10] as well as higher rates of visceral injury [8].

Other factors such as patient profile, the level of preoperative pain, type of hernia, post-operative pain and complications are also being assessed as to their significance in assessing the risk of the development of chronic pain [9]. Many methods including numerical and behavioural rating scales have been used to assess the levels of chronic pain [11], attesting to the difficulty in assessment and interpretation. Standardization of methods of measuring 
results is required [7].

Franneby's [11] validated chronic inguinal pain questionnaire (IPQ) was used in this study. This was chosen because of the comprehensive but simple nature of the questionnaire. This also incorporated pain behavior rather than numbers. The IPQ also addressed many of the issues surrounding this difficult concept, and went a great way towards providing accurate assessment.

Many of the multicentre trials used in larger systematic reviews [8] that govern current guidelines [12] incorporate many different surgeons of varying levels of experience [10]. To gain further insight a consecutive series of patients operated on using the Tension Free Lichtenstein Technique (TFLT) with local anaesthesia and standard mesh in 2005 by a single experienced hernia surgeon were reviewed.

The primary objective of this study was to assess the incidence of chronic pain, using a validated inguinal pain questionnaire [11]. This series aims to address issues previously raised when investigating the incidence of chronic pain [13]. The Lichtenstein technique [14] was used in a consecutive series of patients with unilateral, bilateral and recurrent inguinal hernias.

\section{Methods}

Approval was obtained from The Avenue Hospital Human Research Ethics Committee, Ramsay Health, Melbourne Australia.

\subsection{Patients Selection \& Baseline Data}

All patients who underwent a primary inguinal hernia, bilateral inguinal hernia, or recurrent inguinal hernia repair in 2005 were included. Patients were then contacted in 2009, at a median of 50 months post-operatively by one of the research team. The follow-up rate was $75 \%$. An extensive interview based on Franneby's IPQ [11] was conducted.

A study of the preoperative medical records, together with audit forms completed at the time of operation and review was undertaken. The level of pre-operative pain, co-morbidities and type and size of the hernia had been recorded. The operative findings method of repair, mesh and fixation used, together with the management of the nerves had been documented. The post-operative complications, post-operative pain, patient's interpretation of the pain, and return to normal activities and work had been documented during the routine post-operative visits. The level of analgesics required post-operatively and return to normal activities was reviewed.

\subsection{Inguinal Pain Questionnaire (IPQ)}

The IPQ uniquely explored pain intensity rather than its presence or absence. This allowed for a more meaningful examination of pain, and pain behavior. The IPQ measured:
- Pain and its impact on daily activities across four different periods: preoperatively, post-operatively, time of interview, and the week preceding the interview. The following scale was used:

1) No Pain.

2) Pain present but can easily be ignored.

3) Pain present, cannot be ignored, but does not interfere with everyday activities.

4) Pain present, cannot be ignored, interferes with concentration on chores and daily activities.

5) Pain present, cannot be ignored, interferes with most activities.

6) Pain present, cannot be ignored, necessitates bed rest.

7) Pain present, cannot be ignored, prompt medical advice sought.

- When pain ceased post operatively.

- How often had the participant felt pain in the operate groin during the past week, and how long had it lasted.

- Current analgesia requirements.

- Activities of daily life associated questions.

- Any work limitations.

\subsection{The Lichtenstein Technique [14]}

\subsubsection{Anaesthesia}

All repairs were carried out using Local Anaesthetic (LA) infiltration and light intravenous sedation, including Fentanyl, Propofol or Midazolam and anti-inflammatory agents. The combination used depended largely on the anaesthetists preference. A mixture of Lignocaine $2 \%$ with Adrenaline 1:200,000 and plain Bupivacaine 0.5\% were used. LA was directly infiltrated into the skin and subcutaneous tissues after an initial dose of sedation. The sedation avoided the possible discomfort of the injections. The ilioinguinal nerve (IIN) and the iliohypogatric nerves (IHN) were blocked by introducing the LA deep to the external oblique aponeurosis under direct vision. This gave rapid anaesthesia and displaced the IIN and IHN from the external oblique making direct injury to the nerves and their perineurium less likely. The LA helped identify and dissect the tissue planes as it was injected around the hernial sac and cord and into the region of the genital division of the GFN.

A formal ilio-inguinal nerve (IIN) block at the anterior superior iliac spine was not performed, as in the surgeons experience patients frequently complained of post-operative pain at the site of injection. Moreover, this technique takes longer to become effective and adds to the overall volume of LA required.

\subsubsection{The Nerves}

The identification and management of the nerves was recorded. An attempt was made to identify all 3 nerves. However an extensive search was not carried out as this could increase tissue trauma and possibly damage the 
nerves. In the majority of cases, all nerves were identified and spared. If the nerve had been traumatised or was compromised by the mesh or suturing, it was dissected back to the muscle, divided and removed totally, (neurectomy). Diathermy or ligation of the stump was not employed.

The IIN was usually not separated from the cord. Care was taken in closing the external oblique to avoid entrapping the IIN.

\subsection{Surgical Technique}

The Lichtenstein technique has been well described [14]. Some important aspects of the technique and possible differences include:

- No diathermy was used. The authors believed this could cause tissue and nerve damage setting up a neuropathic and nocioceptive inflammatory response.

- Adrenaline kept the blood loss to a minimum.

- Sharp dissection was used to reduce trauma.

- The Local Anaesthetic technique requires a gentler dissection.

- For indirect hernias the sac was either excised or reduced (especially for sliding hernias).

- For direct hernias the sac was reduced.

- Any additional lipoma of the cord was always excised.

- A standard Polypropylene mesh was used Prolene (trademark) mesh, Polypropylene, non-absorbable synthetic surgical mesh, Johnson \& Johnson.

- A standard skin stapler (Appose $35 \mathrm{w}$ auto suture) was used to fix the mesh to the inguinal ligament as per the Lichtenstein technique. The mesh was placed well medial to pubic tubercle, but the staples were placed well away from the pubic tubercle.

\section{Results}

A total of 248 patients were operated on in 2005. This equated to 283 hernias including 35 bilateral, and 23 recurrences. 185 patients were contacted in 2010, this equated to 213 hernia repairs with 28 bilateral and 16 recurrences equating to a follow-up rate of 75\% (Table 1).

\subsection{Patient Demographics (of Original Cohort)}

Age distribution was between 18 - 90 years. The majority between 50 - 60 years of age $n=73$ (28.85\%). 241 (97\%) of the patients were male, and 7 (3\%) were female.

\subsection{Inguinal Pain Questionnaire (IPQ)}

$67 \%$ ( $n=124$ ) of patients reported pre operative pain. This ranged in severity between pain that could be easily ignored $(27 \%)$ to pain which required hospitalization $(3 \%) .33 \%(n=61)$ of patients reported no pain at all.

The proportion of patients with pain at time of interview was 3\% ( $<<0.001)$ (Table 2$)$. Of those who reported pain:

- $1.1 \%(n=2)$ reported that their pain was short lived, just a twinge and did not interfere with their normal activities could be easily ignored and did not require painkillers.

- $1.6 \%(n=4)$ reported having pain, which did not interfere with their activities but could not be easily ignored (but still not sufficient to require analgesia).

- No patients reported pain that interfered with their daily activities, required analgesia or required medical attention.

The proportion of patients with pain in the week prior to interview (which included those who identified pain at the time of interview) was 5\% $(\mathrm{P}<0.001)$ (Table 2). Of those who reported pain:

- 2.2\% ( $\mathrm{n}=4)$ pain short lived. Easily ignored. Not interfering with normal activities. Analgesics not used.

- $3.2 \%(n=6)$ pain not interfering with activities, but could not be easily ignored (not sufficient to require analgesia).

- 1 patient reported pain that needed regular analgesia but did not interfere with their daily activities. They did not need medical attention.

Table 1. Number of patients, and distribution of hernia subtypes.

\begin{tabular}{cccc}
\hline & & Performed in 2005 & $\begin{array}{c}\text { Analyzed by } \\
\text { follow up 2010 }\end{array}$ \\
\cline { 3 - 4 } Group & Patients & 248 & 185 \\
& Hernias & 283 & 213 \\
& Unilateral hernias & 213 & 157 \\
& Bilateral hernias & 35 & 28 \\
& Recurrent & 23 & 16 \\
\hline
\end{tabular}

Table 2. Comparison of pain ratings.

\begin{tabular}{|c|c|c|c|}
\hline & & Pain right now & Pain in prior week \\
\hline \multirow{7}{*}{ Pain rating } & No pain & $180(97.3 \%)$ & $175(94.6 \%)$ \\
\hline & Pain present, easily ignored & $2(1.1 \%)$ & $4(2.2 \%)$ \\
\hline & Pain present, cannot be ignored but does not interfere with activities & $3(1.6 \%)$ & $6(3.2 \%)$ \\
\hline & Pain present, cannot be ignored and interferes with concentration and activities & $0(0 \%)$ & $0(0 \%)$ \\
\hline & Pain present, interferes with most activities & $0(0 \%)$ & $0(0 \%)$ \\
\hline & Pain present necessitates bed rest now & $0(0 \%)$ & $0(0 \%)$ \\
\hline & Pain present advice sought & $0(0 \%)$ & $0(0 \%)$ \\
\hline
\end{tabular}




\subsection{Resolution of Pain Post-Operatively}

$83 \%(n=154)$ of patients were pain free at 1 month post operatively, and $92 \%(n=170)$ at 2 - 3 months post-operatively (Table 3). Of the other $8 \%$ : $3 \%$ had intermittent pain that lasted for 6 months (not interfering with activities), $4 \%$ of patients experienced pain for up to 12 months (not interfering with activities), 1\% had pain for up to 24 months post-operatively.

\subsection{Post Operative Analgesia Requirements}

Patients were prescribed paracetamol and codeine tablets (500 mg \& $30 \mathrm{mg}$ combination) postoperatively, and were advised to down grade to the $500 \mathrm{mg} / 8 \mathrm{mg}$ combination or the paracetamol $500 \mathrm{mg}$ only preparation as soon as pain allowed or if they were having side effects from the analgesia (Table 4).

\subsection{Functional Status Questions (at Time of Interview and Previous Week)}

- $100 \%(n=185)$ of patients had no pain when getting up from a low chair.

- $97.8 \%(n=180)$ of patients reported no pain when sitting for more than half an hour.

- $98.4 \%$ ( $\mathrm{n}=182$ ) 182of patients did not experience any pain or discomfort when standing for more than half an hour.

- $98.9 \%(n=183)$ of patients were able to go up and down stairs without experiencing any pain in the groin.

- $98.4 \%(n=182)$ had no pain when driving.

\subsection{Complications}

- None of the patients with significant complications developed significant chronic pain or disability.

- One patient re-operated on for bleeding, due to anticoagulation following embolus, had occasional discomfort.

- One patient who needed removal of a staple from the

Table 3. Resolution of pain post operatively. $P$ values calculated when cross-tabulated against preoperative pain.

\begin{tabular}{lccc}
\hline \multicolumn{3}{c}{ Percentage Pain Free } & P Value \\
\hline \multirow{2}{*}{ Time Since Operation } & 1 Month & 83 & $\mathrm{P}<0.001$ \\
& 2 - 3 Months & 92 & $\mathrm{P}<0.001$ \\
\hline
\end{tabular}

Table 4. Post operative analgesia requirements (including patients who were unable to be contacted).

\begin{tabular}{cccc}
\hline & No Analgesia & $\mathrm{n}$ & $\%$ \\
\cline { 3 - 4 } & One Day of Analgesia & 47 & 14.5 \\
Days of Analgesia & $2-3$ Days of Analgesia & 98 & 19.0 \\
& $>49.5$ \\
& Incomplete Data & 52 & 21.0 \\
\hline
\end{tabular}

mesh had no further pain.

- One patient who required prostatectomy had no further pain.

- Continuing audit over many years showed these complications (PE, DVT, acute urinary retention) to be one off events.

- The patients with seromas and superficial infections had no further problems, as did the patients who developed recurrences, which were repaired.

\subsection{Treatment of Nerves}

Although the data was incomplete, the IIN was identified in approximately $80 \%$ of cases. In approximately $10 \%$ of these cases when the nerve was identified a neurectomy was performed. This was carried out either as a result of accidental damage, excessive dissection or the fear of entrapment in the mesh.

The IHN was identified less frequently-in approximately $70 \%$ of cases. It was divided accidently or intentionally in approximately $10 \%$ of these cases mainly to avoid entrapment in fixation of the mesh as it emerged medially from the internal oblique aponeurosis.

The GFN was always identified with the cremasteric vessels and only divided and ligated in a few cases when these vessels were ligated for technical reasons.

\section{Discussion}

The vast majority of unilateral, bilateral or recurrent hernia patients at 50 months had no significant pain or disability. None reported that their exercise, activities or work were limited by pain. Few reported the need for analgesia on any consistent basis. The incidence of moderate or significant chronic pain (that is pain which interfered with activity or required regular analgesia) was less than $1 \%$. In view of the high incidence of chronic pain and disability in some series [10] there have been many attempts to identify possible risk factors and surgical materials and techniques that might predict its development. This study, because of the low incidence of chronic pain was unable to identify any previously reported risk factors, despite the cohort being a consecutive series of patients.

The wide discrepancy in the reported incidence of chronic pain after inguinal hernia repair results needs to be explained particularly as recommendations may be based on these results [12]. The authors here seek a possible explanation for this.

It has been pointed out that aggressive early therapy for post-operative pain is indicated, since the intensity of post-operative pain correlates with the risk of developing chronic pain [15].

Pre-operative LA was used routinely as part of this regime ensuring the patient is pain free for at least $4-10$ hours and is able to travel home in comfort without the 
need for analgesics. It was noted in this series that the vast majority of the patients did not consider early postoperative pain to be a major factor. The use of post-operative analgesics was: $14 \%$ needed no painkillers, $18 \%$ used pain killers for 1day, and the majority for just a few days to a week. Even those who felt post-operative pain to be an issue did not develop significant chronic pain. Those patients who did complain of post-operative pain at one week were kept under review until the pain resolved.

The low incidence of significant early post-operative pain or perceived pain and the minimal need for analgesia in many patients, may be of significance. The LA may contribute to this early low level of pain and may be a significant factor, particularly as pre-emptive, peri-operative and post-operative analgesia considered under the title "multimodal analgesia" are being assessed as factors in preventing chronic pain [16].

Furthermore with LA many of the early side effects of general anaesthesia such as nausea, vomiting, and acute retention of urine are reduced. Less intensive post-operative nursing, including airway care is required. The majority of patients go home within 3 hours of surgery. The long acting LA lasts from 4 - 10 hours and many patients do not need further analgesia. Many patients preferred the LA because of previous problems with general anaesthesia.

Many of the studies of the Lichtenstein method have not used local anaesthesia as described by Lichtenstein. This may diminish the benefits of the original repair, and account for a higher incidence of chronic pain found in some studies [8].

Similarly the role of surgeon experience has been discussed previously, but not yet explored [17].

\subsection{The Nerves}

The management of the 3 major nerves of the inguinal canal has been considered to be a factor in chronic pain [18]. This study showed a low incidence of chronic pain despite the IIN and IHN not being formally identified or damaged and removed in more than $20 \%$ of cases.

Extensive studies concluded that identification and of all 3 nerves of the inguinal canal could influence the incidence of chronic groin pain $[19,20]$.

\subsection{Mesh, Staples}

Mesh and staples have also been widely implicated as significant factors in the development of chronic pain leading to a variety of new lighter weight meshes, staples and glues [16]. This series with its low incidence of significant chronic pain using a standard Polypropylene mesh and non-absorbable staples raises the question as to their role.

\subsection{Positive Results}

The positive results identified in this series may be due to the following factors.

LA infiltration allowing simpler dissection of the tissues with less trauma. Diathermy is not used, possibly reducing the inflammatory response around the nerve endings, a possible cause of nocioceptive pain. Identification and management of the nerves [12]. The use of the open skin stapler to fix the mesh (appose ulc $35 \mathrm{w}$ auto suture). The early supervised management of post-operative pain, including contact by telephone contact by the surgeon for all patients, the day following surgery to adjust analgesia and give support as necessary.

If the results vary so much, is it possible to attribute chronic pain to the mesh/fixation alone? The results in this study, suggest that mesh and staples may not be the main factors in determining the incidence of chronic pain, and could it just be the way the materials are used? Does it depend on the technique and the surgeon?

\section{Conclusions}

There is strong evidence from this series, using a validated inguinal pain questionnaire, that a Lichtenstein repair with local anaesthesia can result in a low incidence of moderate or severe significant chronic post-operative pain. Those few patients who did report pain requiring analgesia, did not have any associated significant morbidity or impairment of activities of daily living. Thus there appeared to be no reason to alter the type of mesh used or its method of fixation. As well the approach taken to the identification and management of the nerves appeared to achieve satisfactory results.

The validated IPQ provides a more detailed appreciation of the pain. This will be useful in the future to help in assessing the role of surgical risk factors, surgical materials, management of the nerves and even the skill of the surgeon as a cause of chronic pain.

\section{Acknowledgements}

Prof. Avni Sali, Prof. Steven Chan, Prof. Julian Smith, Mrs. Di Bloore.

\section{REFERENCES}

[1] A. Kingsnorth and K. LeBlanc, "Hernias: Inguinal and Incisional," Lancet, Vol. 362, No. 9395, 2003, pp. 1561-1571. doi:10.1016/S0140-6736(03)14746-0

[2] T. Bisgaard, et al., "Risk of Recurrence 5 Years or More after Primary Lichtenstein Mesh and Sutured Inguinal Hernia Repair,” British Journal of Surgery, Vol. 94, No. 8, 2007, pp. 1038-1040. doi:10.1002/bjs.5756

[3] H. Kehlet, "Chronic Pain after Groin Hernia Repair," British Journal of Surgery, Vol. 95, No. 2, 2008, pp. 135-136. doi:10.1002/bjs.6111

[4] A. S. Poobalan, et al., "Chronic Pain and Quality of Life Following Open Inguinal Hernia Repair,” British Journal 
of Surgery, Vol. 88, No. 8, 2001, pp. 1122-1126. doi:10.1046/j.0007-1323.2001.01828.x

[5] M. Bay-Nielsen, et al., "Swedish Hernia Data Base the Danish Hernia Data B. Chronic Pain after Open Mesh and Sutured Repair of Indirect Inguinal Hernia in Young Males,” British Journal of Surgery, Vol. 91, No. 10, 2004, pp. 1372-1376. doi:10.1002/bjs.4502

[6] R. E. Condon, “Groin Pain after Hernia Repair,” Annals of Surgery, Vol. 233, No. 1, 2001, p. 8. doi:10.1097/00000658-200101000-00002

[7] H. Kehlet, et al., "Chronic Postherniorrhaphy Pain-A Call for Uniform Assessment,” Hernia, Vol. 6, No. 4, 2002, pp. 178-181. doi:10.1007/s10029-002-0082-0

[8] K. McCormack, et al., "Laparoscopic Techniques versus Open Techniques for Inguinal Hernia Repair,” Cochrane Database of Systematic Reviews, Vol. 2003, No. 1, 2003, Article ID: CD001785.

[9] K. J. Dickinson, et al., "Predicting Chronic Post-Operative Pain Following Laparoscopic Inguinal Hernia Repair,” Hernia, Vol. 12, No. 6, 2008, pp. 597-601. doi:10.1007/s10029-008-0408-7

[10] H. R. Langeveld, et al., "Total Extraperitoneal Inguinal Hernia Repair Compared with Lichtenstein (the LEVELTrial): A Randomized Controlled Trial," Annals of Surgery, Vol. 251, No. 5, 2010, pp. 819-824. doi:10.1097/SLA.0b013e3181d96c32

[11] U. Franneby, et al., "Validation of an Inguinal Pain Questionnaire for Assessment of Chronic Pain after Groin Hernia Repair,” British Journal of Surgery, Vol. 95, No. 4, 2008, pp. 488-493. doi:10.1002/bjs.6014

[12] M. P. Simons, et al., "European Hernia Society Guidelines on the Treatment of Inguinal Hernia in Adult Patients," Hernia, Vol. 13, No. 4, 2009, pp. 343-403. doi:10.1007/s10029-009-0529-7

[13] U. Franneby, et al., "Risk Factors for Long-Term Pain after Hernia Surgery,” Annals of Surgery, Vol. 244, No. 2, 2006, pp. 212-219.

doi:10.1097/01.sla.0000218081.53940.01

[14] S. Lichtenstein, et al., "The Tension Free Hernioplasty," American Journal of Surgery, Vol. 157, 1989, pp. 188193. doi:10.1016/0002-9610(89)90526-6

[15] H. Kehlet, et al., "Persistent Postsurgical Pain: Risk Factors and Prevention,” Lancet, Vol. 367, No. 9522, 2006, pp. 1618-1625. doi:10.1016/S0140-6736(06)68700-X

[16] E. K. Aasvang, et al., "Predictive Risk Factors for Persistent Postherniotomy Pain,” Anesthesiology, Vol. 112, No. 4, 2010, pp. 957-969. doi:10.1097/ALN.0b013e3181d31ff8

[17] C. G. Schmedt, et al., "Comparison of Endoscopic Procedures vs. Lichtenstein and Other Open Mesh Techniques for Inguinal Hernia Repair: A Meta-Analysis of Randomized Controlled Trials,” Surgical Endoscopy, Vol. 19, No. 2, 2005, pp. 188-199. doi:10.1007/s00464-004-9126-0

[18] K. Caliskan, et al., "A Method for the Reduction of Chronic Pain after Tension-Free Repair of Inguinal Hernia: Iliohypogastric Neurectomy and Subcutaneous Transposition of the Spermatic Cord," Hernia, Vol. 14 No. 1, 2010, pp. 51-55. doi:10.1007/s10029-009-0571-5

[19] J. F. M. Lange, et al., "Prophylactic Ilioinguinal Neurectomy in Open Inguinal Hernia Repair: A Double-Blind Randomized Controlled Trial,” Annals of Surgery, Vol. 246, No. 6, 2007, p. 1116. doi:10.1097/SLA.0b013e31815c2979

[20] S. Alfieri, et al., "Influence of Preservation versus Division of Ilioinguinal, Iliohypogastric, and Genital Nerves during Open Mesh Herniorrhaphy: Prospective Multicentric Study of Chronic Pain,” Annals of Surgery, Vol. 243, No. 4, 2006, pp. 553-558. doi:10.1097/01.sla.0000208435.40970.00 